\title{
Conductance quantization and backscattering
}

\author{
Jens U. Nöckel* \\ I. Institut für Theoretische Physik \\ Universität Hamburg, Jungiusstrasse 9 \\ D-2000 Hamburg 36 \\ Federal Republic of Germany \\ Published in Physical Review B 45 (24), 14225 (1992)
}

(Received 6 January 1992)

The transmission behaviour of a quantum point contact in a magnetic field is calculated, exhibiting in detail the influence of intersubband scattering. In contrast to the commonly known reduction of backscattering by a magnetic field, it is shown here that a weak field can cause increased backscattering. Nevertheless, the present results serve to illustrate that backscattering can occur without any effect on the conductance quantization, which is explained by an indirect compensation mechanism.

\section{Introduction}

It was recently discovered by VAN WEES ET AL. [17] and WhARAM ET AL. [18] that the conductance of a nanostructured constriction in a two-dimensional electron gas (referred to as quantum point contact, QPC) exhibits steps of magnitude $2 \frac{e^{2}}{h}$ if the constriction width is varied. In the modulation-doped GaAs-AlGaAs heterostructures used to perform the experiments, the spatial extension of the depletion regions defining the QPC can be varied by changing a gate voltage $V_{g}$. At the origin of the quantized conductance is the formation of one-dimensional channels in the device:

When $B=0$, one-dimensional modes are formed because the confining potential quantizes electronic motion perpendicular to the device walls. At moderate magnetic fields, these modes become hybrid channels, or magneto-electric subbands, due to the simultaneous presence of spatial and Landau quantization. In a high magnetic field, the one-dimensional subbands take the form of edge channels in which an electron propagates coherently along spatially separated equipotential lines [12].

At any point in the constriction, these subbands appear as discrete branches of the dispersion relation $E$ versus $k$, where $k$ is the wavenumber associated with the direction of free motion (choosing Landau gauge at $B \neq 0$ ).

\footnotetext{
${ }^{*}$ Current address: Department of Physics, University of Oregon. http://uoregon.edu/ noeckel
} 
As the height and width of the saddle shaped depletion potential are varied, a subband will cease to contribute to the current when it is pushed above the Fermi energy at the narrowest point of the constriction. Since each of the onedimensional subbands contributes $2 \frac{e^{2}}{h}$ to the conductance $G$ due to a cancellation between density of states and average velocity [2], there will be a step in $G$ whenever the width $W$ reaches the cutoff-value for the the highest subband. Experimentally observed departures from such accurate steps in the conductance can be attributed to the presence of barrier penetration, above-barrier reflection and intersubband scattering.

The effects of these complications on the accuracy of the conductance steps have been investigated theoretically by several authors, using various model potentials $[14,19-25]$.

To translate scattering properties of the QPC into the conductance measured between the $2 \mathrm{D}$ reservoirs, one can make use of the two-terminal Landauer-type conductance formula derived in [5]. The conductance is given in units of $\frac{e^{2}}{h}$ by

$$
g=\sum_{a, b}\left|T_{b a}\right|^{2},
$$

where $T_{b a}$ is the current transmission amplitude from subband $a$ to $b$, and electron spin is not considered, as in [5]. The simplest way to obtain well-defined steps in the conductance versus constriction width is to assume $T$ diagonal and the diagonal elements either of magnitude one, for channels propagating through the constriction, or zero for channels that are not. This means if $W$ is the constriction width, $\left|T_{a a}(W)\right|^{2}$ must be step functions in $W$, a condition that is met whenever the simple introductory explanation above holds where resonances, tunneling and subband mixing are not considered. For intersubband scattering to be absent, the transport is required to be adiabatic, this being well satisfied in the edge channel regime. At $B=0$, the electronic motion is generally not one-dimensional, because scattering between subbands can easily be caused by the device geometry [22] as well as by disorder [24].

$T$ is indeed diagonal for the model potential treated by BüTTIKER [23]. However, as is also stated in [23], conductance steps can still be expected if $T$ is not diagonal. BÜTTIKER's argument is that any diagonal $T$ that yields steps in (1) can be subjected to an arbitrary unitary transformation which creates a non-diagonal $T^{\prime}$ while leaving the result of (1) unaffected. A physical interpretation for this can be given by pointing out with BEENAKKER, VAN HOUTEN [2] that conductance quantization may persist as long as intersubband scattering does not alter the net transmission behaviour of a QPC, which is all that is left after the summation in (1).

A special case of a non-diagonal $T$ has been discussed by LAUGHTON et al. [24]: They find quantized transmission in the presence of impurities, povided that only forward scattering takes place. The reason for this was given by PAYNE [19], who pointed out that intersubband scattering has no effect on the conductance steps as long as scattering from subband $a$ to subband $b$ is exactly compensated by the opposite process from $b$ to $a$. In that case, channel $a$ carries the same current before and after the obstacle until the subband cutoff threshold is reached, at which time the conductance has a step. For $B=0$, it is argued in [19] that this cancellation of scattering events occurs if both channels are fully populated up to the Fermi energy. The latter can be satisfied for all except the reflecting channels, so that the conductance remains quantized when the scattering is purely forward.

Writing the above compensation argument in terms of transmission coefficients, the absence of significant backscattering means that the net current each channel 
sustains only depends on whether or not the channel is conducting at all, so that for all $b$

$$
\sum_{a}\left|T_{b a}(W)\right|^{2}=\Theta\left(W-W_{b}\right),
$$

where $W_{b}$ in the step function $\Theta$ is the cutoff width below which no current can emerge on the far side of the constriction in final state $b$. Since $\left|T_{a b}\right|^{2}=\left|T_{b a}\right|^{2}$, we can exchange the indices in (2) and thus obtain as an equivalent statement

$$
\sum_{b}\left|T_{b a}(W)\right|^{2}=\Theta\left(W-W_{a}\right)
$$

for all $a$. This formula means that the incoming current in each individual subband $a$ only branches into the available outgoing channels, so that the net transmission is unity as long as subband $a$ is not cut off.

As was to be expected from the reasoning of PAYNE, a lowering of the conductance plateaux due to backscattering is also found in [24], as well as in [21]. In the presence of a magnetic field, conductance quantization generally becomes more accurate due to the reduction of backscattering from impurities [8] as well as from the constriction geometry [2, 14], and the conductance plateaux broaden because the subband separation increases.

Guided by the very general, but abstract argument of BüTTIKER, we now ask whether a more general $T$ than the one in (2) could also be physically realized. Put differently, can there be quantization of the conductance if backscattering is important so that (2) does not hold? The present calculation proves that this question can be answered affirmatively.

Very recently, a recursive Green's function calculation has been performed by ANDO [25], yielding results very similar to ours in spite of the completely different computational method employed here. However, we show that the action of a weak magnetic field in the non-adiabatic regime cannot be interpreted in terms of the commonly known reduction of backscattering. To the contrary, backscattering can actually be increased by the magnetic field while conductance quantization improves at the same time. The reason for this is a modified current compensation process that allows for backscattering. This is in contrast to the results quoted above, which seem to suggest that backscattering is invariably detrimental to the accuracy of conductance quantization.

The reason why this effect of a magnetic field cannot be seen in the work done by ANDO will be explained in the next section.

\section{Model}

At $B=0$, one can use the fact that the time independent Schrödinger equation for the ballistic electron motion is formally identical to the Helmholtz equation of waveguide optics. One characteristic of this "electron optics" is that in the constriction, any potential barrier that depends on the longitudinal $(x)$ coordinate alone can cause no scattering between different channels [22], since a Schrödinger equation of the type

$$
\left\{\frac{p_{x}^{2}}{2 m}+\frac{p_{y}^{2}}{2 m}+V^{\prime}(x)+V(y)\right\} \Psi(x, y)=E \Psi(x, y)
$$

is separable.

However, an additional vector potential destroys the formal analolgy to optical waveguides. This becomes significant in weak magnetic fields, where the magnetic 
length is comparable to a characteristic length of the potential variation, so that neither the waveguide nor the semiclassical skipping orbit pictures are valid. Under these circumstances, the magnetic field itself becomes a third independent source of subband mixing besides device geometry and impurities, as will now be illustrated by introducing a vector potential in equation (4): If the barrier $V_{x}$ is abrupt, subband conservation will only hold in $B=0$, but not in $B \neq 0$, because there is no choice of gauge or coordinate system for which the Schrödinger equation

$$
\left\{\frac{1}{2 m}\left(\vec{p}+\frac{e}{c} \vec{A}(x, y)\right)^{2}+V^{\prime}(x)+V(y)\right\} \Psi(x, y)=E \Psi(x, y)
$$

separates for a rectangular barrier $V^{\prime}(x)$. Such magnetic-field induced subband mixing will be investigated here.

Following BÜTTIKER [23], we approximate the transverse confinement by a quadratic potential

$$
V(y)=\frac{1}{2} m \omega_{0}^{2} y^{2}
$$

Here, $\omega_{0}$ does not vary with $x$ because we do not consider scattering at the interfaces between reservoirs and QPC (see [2, 4, 22]). Into this quantum wire which represents the constriction region, we now introduce an additional barrier in the form of a multistep function $V_{b}(x)$ in the longitudinal direction. Thus we obtain the saddle-shaped potential landscape of a realistic QPC by piecing together several identical parabolic wire segments with a bottom offset of $V_{b}$.

In our model, to increase $V_{b}$ is to change two things at the same time in the classical potential landscape seen by an electron at the fermy energy: rising barrier height is accompanied by a narrowing of the constriction at $E_{F}$. The advantage of this geometry is that it guarantees subband conservation for $B=0$, irrespective of how abrupt the potential variations are, cf. (4). In ANDO's treatment [25] a magnetic field is never the sole cause of intersubband scattering since subbands are mixed even at $B=0$. Due to its simplicity, the present model allows to separate the effects of a magnetic field on subband conservation from the effects of the device geometry. The amount of intersubband scattering can be determined solely by the magnetic field strength, without having to change the scattering potential. This feature cannot be exploited if the saddle potential is continuous and slowly varying since such a $V_{b}(x)$ entails adiabatic transport independently of $B$. Therefore, it is appropriate to consider not a smooth, but an abrupt potential.

\section{Calculations}

The electronic motion in our model potential will be found by performing a wave function matching similar to that in $[20,26]$ but generalized to the case $B \neq 0$.

Expressing the magnetic field in terms of the cyclotron frequency $\omega_{c}=\frac{e B}{m c}$, and choosing the Landau gauge $\vec{A}=-B y \vec{e}_{x}$, our starting point is the Hamiltonian

$$
H=\frac{1}{2 m}\left\{\left(p_{x}-m \omega_{c} y\right)^{2}+p_{y}^{2}\right\}+\frac{1}{2} m \omega_{0}^{2} y^{2}+V_{x}
$$

with a constant potential $V_{x}$ and an oscillator frequency $\omega_{0}$ independent of $x$. To introduce dimensionless variables, we define a hybrid frequency, $\omega:=\sqrt{\omega_{0}^{2}+\omega_{c}^{2}}$,

and measure frequencies in units of $\omega$, energies in $\hbar \omega$, and lengths in $\sqrt{\frac{\hbar}{m \omega}}$. In these units one has $\omega_{0}^{2}+\omega_{c}^{2}=1$. 
The eigenfunctions, normalized to unit 2D current, have the form

$$
\Psi(x, y)=\sqrt{\frac{1}{|k|}} e^{i k x} u_{a}\left(y-\omega_{c} k\right)
$$

where

$$
u_{a}\left(y-\omega_{c} k\right):=\frac{1}{\sqrt{\sqrt{\pi} a ! 2^{a}}} e^{-\frac{1}{2}\left(y-\omega_{c} k\right)^{2}} H_{a}\left(y-\omega_{c} k\right) \quad(a=0,1, \ldots),
$$

are the harmonic oscillator wavefunctions displaced from the origin by $\omega_{c} k$.

For fixed energy $E$ the wave numbers corresponding to a possible solution are

$$
k_{a}= \pm \frac{\sqrt{2\left(E-V_{x}-a\right)-1}}{\omega_{0}} .
$$

This means that the index of the highest propagating subband is

$$
a_{\max }=\operatorname{int}\left[E-V_{x}-\frac{1}{2}\right] \text {. }
$$

We now relax the assumption $V_{x}=$ const, allowing $N$ discrete discontinuities along the $x$-direction at positions $x_{1}, x_{2}, \ldots, x_{N}$. Let the constant potential in the $N+1$ resulting regions have values $V_{0}, V_{1}, \ldots, V_{N}$. Then in region $\alpha=0 \ldots N$, the wave number belonging to channel $a$ is

$$
k_{a}^{\alpha}= \pm \frac{\sqrt{2\left(E-V_{\alpha}-a\right)-1}}{\omega_{0}} .
$$

We see from (12) and (8) that unless $B=0$, the wavefunction is shifted by unequal amounts in intervals with different $V_{\alpha}$. This mismatch means that an electron incident in subband $a$ from the left will have to evolve into a linear combination of all possible subbands when it crosses $x_{1}$. Likewise, the reflected wave is shifted in the direction opposite to that of the electron incident in $a$, so that the reflection, too, violates subband conservation due to the mismatch of the transverse wavefunctions.

To perform the wave function matching at the discontinuities of $V_{x}$, the displaced transverse functions are expanded in the complete set of the unshifted oscillator eigenfunctions:

$$
u_{a}(y-\Delta)=\sum_{b} c_{b a}(\Delta) u_{b}(y)
$$

the coefficients being [16]

$$
\begin{aligned}
c_{b a}(\Delta) & =\int_{-\infty}^{\infty} u_{b}(y) u_{a}(y-\Delta) d y \\
& =\frac{1}{\sqrt{a ! b ! 2^{a+b}}} e^{-\left(\frac{\Delta}{2}\right)^{2}} \times \begin{cases}2^{a} b !\left(-\frac{\Delta}{2}\right)^{a-b} L_{b}^{a-b}\left(\frac{\Delta^{2}}{2}\right) & (a \geq b) \\
2^{b} a !\left(\frac{\Delta}{2}\right)^{b-a} L_{a}^{b-a}\left(\frac{\Delta^{2}}{2}\right) & (a \leq b)\end{cases}
\end{aligned}
$$

where both cases coincide for $a=b$, and $L_{a}^{b-a}(z)$ denote associated Laguerre polynomials (normalized to $L_{0}^{n}(z)=1$ ).

Using the linear independence of the set $u_{a}(y)$ and requiring the continuous differentiability of the matched wavefunction, one obtains a system of linear equations, the unknowns being the amplitudes $A_{b a}^{\alpha \pm}$ with which subbands $b$ are present in the 


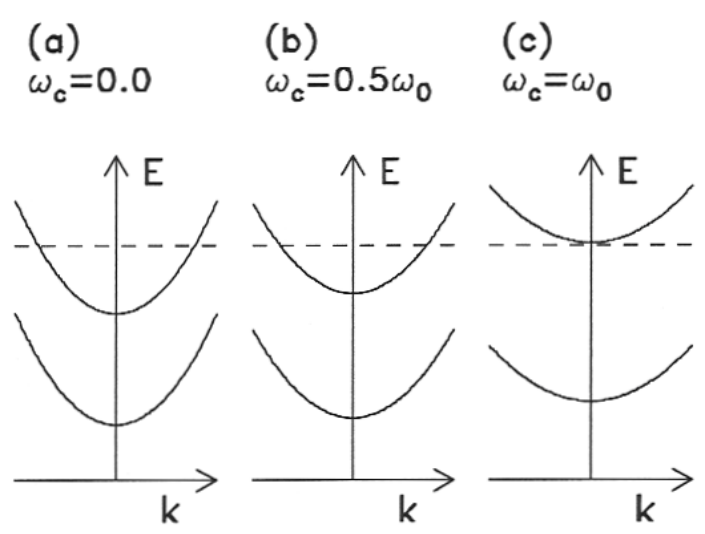

Figure 1: Sketch of the dispersion relation in the regions without barrier potential, for three values of the magnetic field. $E_{F}$ (indicated by the broken line) is chosen so that the second subband becomes depopulated just below $\omega_{c}=\omega_{0}$. The effect of a potential $V_{b}$ is to rigidly shift all the parabolas upward by an amount $V_{b}$. When a subband gets pushed above $E_{F}$ due to $V_{b}$, it no longer propagates in the barrier region.

right- $(+)$ and left-going $(-)$ wave in region $\alpha$ when the electron is incident from the left in subband $a$. This linear system is infinite dimensional because imaginary wavenumbers have to be retained, but it can be well approximated by a system of finite dimension as long as the Lorentz displacement $\omega_{c} k_{a}^{0}$ of the incoming wave is sufficiently small. To arrive at a solution, a standard linear equation solver was used. The system of equations becomes numerically singular if the barrier potential is so high or broad as to decouple the left and right sides. These restrictions were taken into consideration in our choice of parameters.

The quantities of interest are the current transmission and reflection probabilities $\left|T_{b a}\right|^{2}$ and $\left|R_{b a}\right|^{2}$, satisfying [27]

$$
\sum_{b}\left\{\left|R_{b a}\right|^{2}+\left|T_{b a}\right|^{2}\right\}=1
$$

for all $a$. Using arguments similar to those in $[5,6]$, it follows that for our choice of normalisation $T_{b a}=A_{b a}^{N+}$ and $R_{b a}=A_{b a}^{0-}$. The sum rule (14) is used to check the numerical accuracy of the results below.

\section{Results}

We turn now to the simplest case of a rectangular barrier of length $l$ and height $V_{b}$, i.e. $N=2$ and $V_{0}=V_{2}=0, V_{1}=V_{b}$. In a real device, the distance between the inversion layer and the patterned electrodes defining the wire and barrier will determine whether or not such an abrupt conduction band variation can be created. The assumption of an abrupt potential will be unjustified at high magnetic fields because variations in $V^{\prime}(x)$ will always be smooth on the scale of the small magnetic length. Therefore, weak magnetic fields will be investigated here, as in [25]. This means that the edge channel picture is invalid, which is why the magnetic field should no longer be expected to reduce backscattering in the way it is described in $[2,8,14]$.

In zero magnetic field, transverse mode indices $a$ are conserved and transmission probabilities for mode $a$ are [28]

$$
\left|T_{a a}\right|^{2}=\frac{4 k^{2}|\kappa|^{2}}{\left|\left(k^{2}+\kappa^{2}\right) \sin \kappa l+2 i \kappa k \cos \kappa l\right|^{2}},
$$




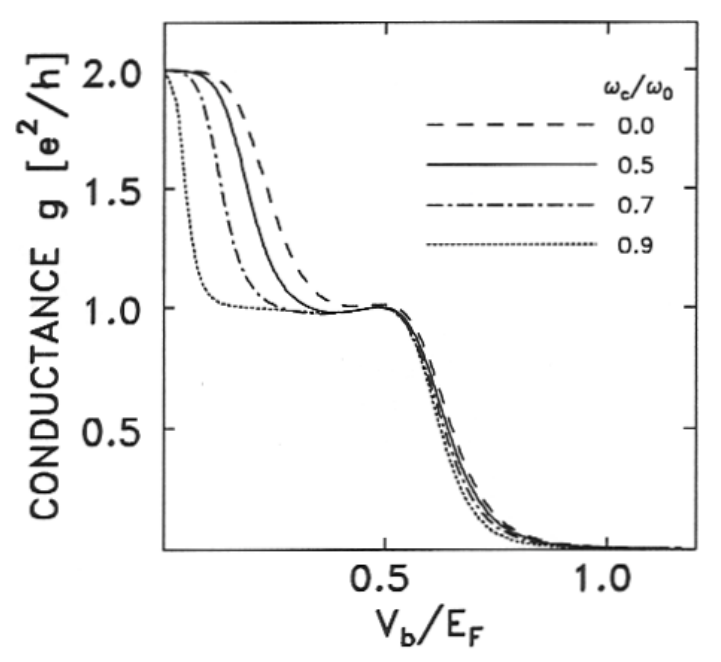

Figure 2: Conductance g as a function of normalized barrier height $\frac{V_{b}}{E_{F}}$ for four values of the magnetic field.

with $k=\sqrt{2(E-a)-1}$ and $\kappa=\sqrt{2\left(E-V_{b}-a\right)-1}$ in reduced units. Our calculation is an extension of this to the case $B \neq 0$. Up to now, the calculations were most compactly written in units that depend on the magnetic field through $\omega$. To be able to compare the results at different values of $B$, all quantities are now expressed in terms of $\omega_{0}$ instead. After converting to these units, expression (11) for the highest propagating subband at a given energy must be modified to

$$
a_{\max }=\operatorname{int}\left[\frac{E-V_{b}}{\sqrt{1+\omega_{c}^{2}}}-\frac{1}{2}\right] .
$$

The dispersion relation in the regions where $V_{b}=0$ is shown in Fig. 1 for three values of $\omega_{c}$ that will now be investigated more closely.

We fix the Fermi energy at $E=2.1 \hbar \omega_{0}$ and the barrier length at $l=3.0 \sqrt{\frac{\hbar}{m \omega_{0}}}$. Consider first the case $\omega_{c}=0$ where, according to Fig. 1(a), the two channels $a=0,1$ propagate outside the barrier. As $V_{b}$ is increased, the conductance exhibits a step which is only approximately at the quantized value $g=1$ of the dimensionless conductance, cf. Fig. 2.This is due to transmission resonances and tunneling, as discussed in [2]. The plateau in conductance vs. $V_{b}$ deviates less from $g=1$ when a magnetic field is applied, and it broadens with increasing field, as the plots for $\omega_{c}=0.5 \omega_{0}$ to $\omega_{c}=0.9 \omega_{0}$ in Fig. 2 show.

At a magnetic field such that $\omega_{c}=\omega_{0}$, Fig. 1(c) tells us that only the $a=0$ subband is still below the Fermi energy, which means the electron is forced to obey subband conservation as in zero field. If we now look at Fig. 3, it is clear that compared to $\omega_{c}=0$, the transmission characteristic $\left|T_{00}\right|^{2}$ has been improved in two ways at $\omega_{c}=\omega_{0}$ : The resonance in the plateau region is damped due to the dephasing action of the magnetic field. Also, tunneling is reduced because some of the longitudinal energy has been drained away into the transverse motion by the magnetic field. This is also the reason why the drop in transmission occurs at a slightly lower potential height when $\omega_{c}=\omega_{0}$.

Having recognized the beneficial effects of a magnetic field on the transmission step in the absence of intersubband scattering, we now introduce a second subband by decreasing the field to $\omega_{c}=0.5 \omega_{0}$. If subband mixing remained absent, no significant change in $\left|T_{00}\right|^{2}$ would be expected. However, Fig. 3 shows that $\left|T_{00}\right|^{2}$ 


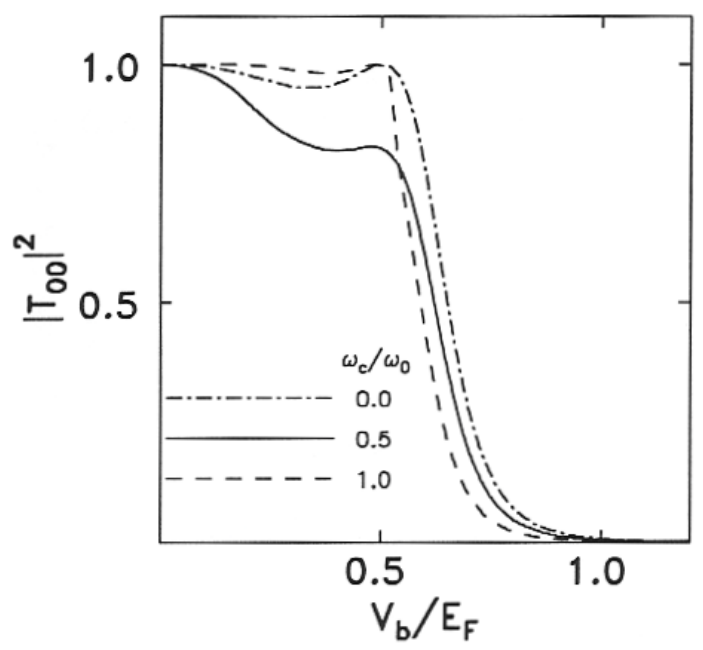

Figure 3: Diagonal current transmission $\left|T_{00}\right|^{2}$ as a function of normalized barrier height $\frac{V_{b}}{E_{F}}$ for three values of the magnetic field. Effects of intersubband scattering are seen at $\omega_{c}=0.5 \omega_{0}$.

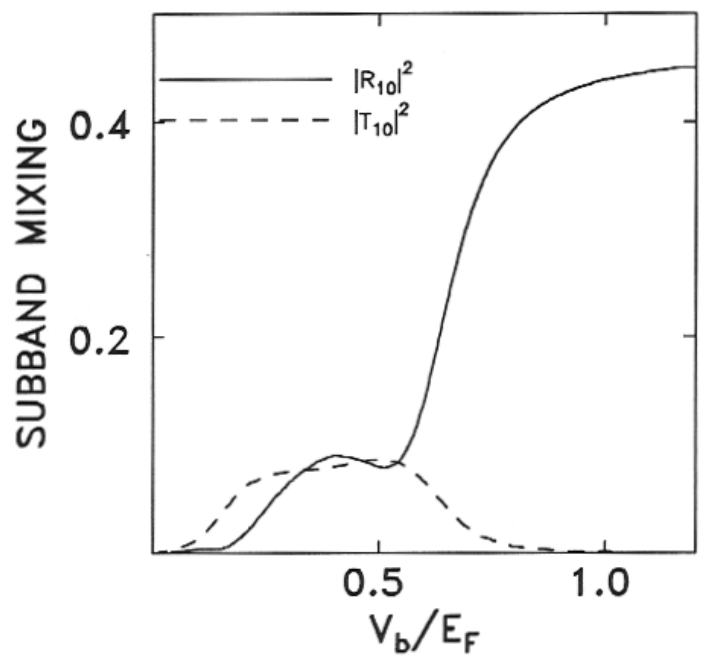

Figure 4: Off-diagonal current reflection $\left|R_{10}\right|^{2}$ and transmission $\left|T_{10}\right|^{2}=\left|T_{01}\right|^{2}$ as a function of normalized barrier height $\frac{V_{b}}{E_{F}}$ at $\omega_{c}=0.5 \omega_{0}$. Reflection occurs before the cutoff potential is reached; for $V_{b} \rightarrow \infty$, an asymptotic value is quickly approached.

loses its step shape at $\omega_{c}=0.5 \omega_{0}$, indicating that a qualitative change has taken place with respect to the purely one-subband cases.

The most striking observation can be made in Fig. 4 which shows that significant off-diagonal reflection occurs in the range of $V_{b}$ where the conductance plateau exists. In addition, a plot of the off-diagonal transmission $\left|T_{10}\right|^{2}$ in the same figure shows that intersubband mixing occurs in the forward direction, too.

Recalling Fig. 2, we can conclude that the conductance quantization is unaffected by intersubband scattering in the forward and backward direction.

A more detailed statement can be made if we inspect $\left|T_{1 a}\right|^{2}+\left|T_{0 a}\right|^{2}$ for $a=0,1$, shown in Fig. 5. The plot for $a=0$ shows that a step shape is not fully recovered due to the substantial backscattering, so that (2) and (3) do not hold.

It follows that the mechanism for conductance quantization must be such that the loss in net transmission of incoming channel $a=0$ is compensated for by an increase in net transmission of channel $a=1$ in the same range of $V_{b}$. This is exactly 


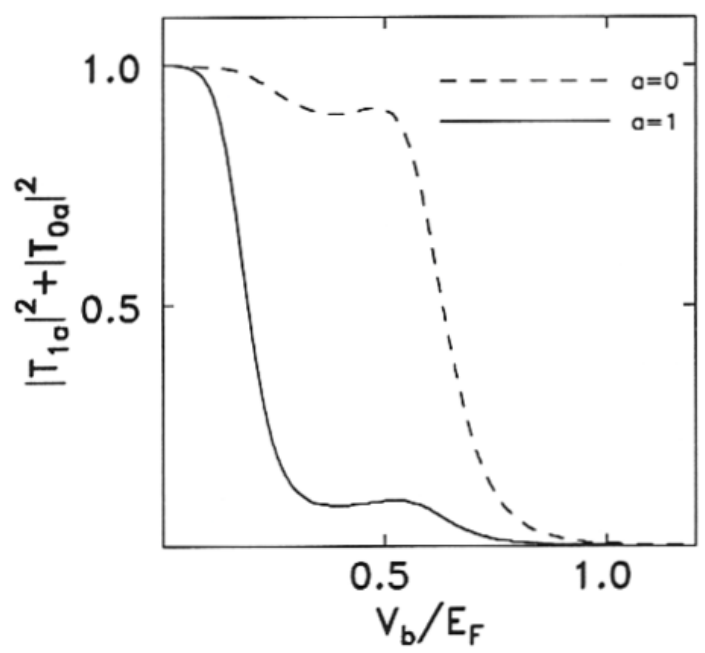

Figure 5: Net current transmission $\left|T_{0 a}\right|^{2}+\left|T_{1 a}\right|^{2}$ as a function of normalized barrier height $\frac{V_{b}}{E_{F}}$ for $a=0,1$ at $\omega_{c}=0.5 \omega_{0}$. In the plateau region, the $a=1$ subband contributes to the current even though it is cut off.

what we see in the net transmission from channel $a=1$, Fig. 5: A transmission shoulder appears in a range of $V_{b}$ where no transmission could occur if channel number was conserved, since the $a=1$ subband is cut off by the barrier. The physical reason why this compensating transmission enhancement can take place is that an incoming electron in channel $a=1$ will be forced to "turn the corner" at the barrier if $V_{b}$ exceeds the cutoff value. While being thus localized near the interface, a transition to $a=0$ can take place, allowing the electron to propagate forward across the barrier before it reaches the reflecting edge. Consequently, the transmission shoulder is essentially $\left|T_{01}\right|^{2}$ since $\left|T_{11}\right|^{2}$ is small when only barrier penetration makes a contribution.

One question that has to be answered to make contact with the compensation argument of [19] is whether all forward scattering events continue to be cancelled by the reverse processes even in the absence of time-reversal symmetry caused by the magnetic field. This can indeed be verified by defining an antiunitary operation $W:=K U$, where $K$ denotes complex conjugation and $U$ is a reflection at the $y$-axis, i.e. $x \rightarrow-x$. The Hamiltonian commutes with $W$, provided the barrier potential is chosen to be symmetric, $V_{b}(x)=V_{b}(-x)$. This fact is sufficient to prove

$$
\left|T_{m n}\right|^{2}=\left|T_{n m}\right|^{2} .
$$

Analogously, one can deduce $\left|R_{m n}\right|^{2}=\left|R_{n m}\right|^{2}$ from the symmetry $V(y)=V(-y)$. However, this property alone does not explain the quantized conductance, because we have to take backscattering into account. In fact, an asymmetric barrier, $V_{b}(x) \neq$ $V_{b}(-x)$, continues to yield a conductance similar to Fig. 2, so that the compensation mechanism (17) is not a relevant explanation at all.

The increased reflection that causes $\left|T_{10}\right|^{2}+\left|T_{00}\right|^{2}$ to deviate from step shape in Fig. 5 is balanced in magnitude by $\left|T_{01}\right|^{2}$. This is due to the peculiar fact that in the range of $V_{b}$ where channel $a=1$ is cut off while $a=0$ propagates in the barrier region, one has

$$
\left|R_{10}\right|^{2} \approx\left|T_{01}\right|^{2}
$$

This can be called an indirect compensation mechanism, and it is not based on a rigorous symmetry argument like that leading to (17). The numerical result reflected 
in Fig. 4 and Eq. (18) can be given a qualitative physical interpretation in the following way: When the magnetic field causes intersubband transitions, it tends to do so with roughly the same strength for both directions of propagation. Thus, the appearance of extra reflection $\left|R_{10}\right|^{2}$ is accompanied by the opening up of an equally effective transmission channel $\left|T_{01}\right|^{2}$. This cooperative effect is suppressed when $V_{b}$ exceeds the cutoff height for the last propagating subband, $a=0$, because in that case $\left|R_{10}\right|^{2}$ grows further while $\left|T_{01}\right|^{2}$ must go to zero. With this result, we have extended the concept of compensation [19] to include cases where backscattering is significant in one channel but masked by enhanced transmission in another.

Finally, a remark must be made on the parameters chosen in this example. The compensation mechanism described here for $\omega_{c}=0.5 \omega_{0}$ works analogously at all magnetic fields for which two subbands are below $E_{F}$ outside the barrier, i.e. between $\omega_{c}=0$ and $\omega_{c}=0.98 \omega_{0}$ (cf. Eq. 16, Fig. 1). This is why all graphs in Fig. 2 exhibit a conductance plateau. The parameter $\omega_{c}=0.5 \omega_{0}$ treated in the detailed analysis corresponds roughly to the magnetic field at which intersubband scattering is at a maximum, because subband index must again be conserved in the limiting cases $\omega_{c} \rightarrow 0$ and $\omega_{c} \rightarrow 0.98 \omega_{0}$.

In the example of [25], the ratio $\frac{\hbar \omega_{c}}{E_{F}}=0.25$ is almost the same as in this work, but for a Fermi energy at which a third subband just propagates at the narrowest point of the QPC when the barrier has zero height. It can be inferred from [25] and my calculations that the indirect compensation mechanism continues to function when more than two subbands are below $E_{F}$.

\section{Conclusion}

The wave-function matching applied in [20] has been generalized to allow for the presence of a magnetic field, resulting in quantitative data on the transmission properties of a QPC in weak magnetic fields. Since the model geometry considered here is separable in the absence of a magnetic field, all intersubband reflection is B-induced.

The fact that conductance quantizaton in a nanostructured constriction persists in the presence of intersubband scattering cannot always be interpreted in terms of a mere redistribution in the forward direction of the net current each incoming channel carries. The net transmission behaviour of our barrier for individual channels does change in a magnetic field, as is seen in particular in the occurence of significant intersubband reflection for a subband that is not cut off by the barrier. In spite of this backscattering, well-defined conductance steps are observed, which is in contrast to the negative role backscattering plays in previous calculations. The mechanism that leads to conductance quantization has been identified as an indirect current compensation process, in which two incoming channels cooperate to form a steplike conductance despite the presence of backscattering. When plateaux in the conductance of a QPC are observed, it is therefore not justified a priori to assume that backscattering is absent in all propagating subbands.

Note added in proof. After submission of this paper, a Rapid Communication by Castaño and Kirczenow appeared [29], giving another convincing example that intersubband scattering can be a decisive factor in the formation of quantized conductance steps. 


\section{References}

[1] D.G. Ravenhall et al., Superlatt. Microstr. 11, 69 (1992)

[2] C.W.J. Beenakker, H. van Houten, Solid State Phys. 44(1991)

[3] J.J. Harris et al., Rep. Prog. Phys. 52, 1217 (1989)

[4] R. Landauer, Z.Phys. 68, 217 (1987)

[5] D.S. Fisher, P.A. Lee, Phys. Rev. B 23, 6851 (1981)

[6] A.D. Stone, A. Szafer, IBM J. Res. Develop. 32, 384 (1988)

[7] A. Messiah, Quantenmechanik Band 1, Walter de Gruyter (1976)

[8] M. Büttiker, Phys. Rev. B 38, 9375 (1988)

[9] J.R. Taylor, Scattering Theory, Wiley (1972)

[10] H.U. Baranger, Phys. Rev. B 42, 11479 (1990)

[11] K.F. Berggren, G. Roos, H. van Houten, Phys. Rev. B 37, 10118 (1988)

[12] B.J. van Wees et al., Phys. Rev. Lett. 62, 2523 (1989)

[13] U. Sivan, Y. Imry, C. Hartzstein, Phys. Rev. B 39, 1242 (1989)

[14] L.I. Glazman, M. Jonson, J. Phys. Condens.Matter 1, 5547 (1989)

[15] J.K. Jain, S.A. Kivelson, Phys. Rev. Lett. 60, 1542 (1988)

[16] I.S. Gradshteyn, I.M. Ryzhik, Table of Integrals, Series and Products, Academic Press, New York (1980)

[17] B.J. van Wees et al., Phys. Rev. Lett. 60, 848 (1988)

[18] D.A. Wharam et al., J.Phys.C 21, L209 (1988)

[19] M.C. Payne, J. Phys.: Condens. Matter 1, 4943 (1989)

[20] A. Szafer, A.D. Stone, Phys. Rev. Lett. 62, 300 (1989)

[21] E.G. Haanappel, D. van der Marel, Phys. Rev. B 39, 5484 (1989)

[22] A. Yacoby, Y. Imry, Phys. Rev. B 41, 5341 (1990)

[23] M. Büttiker, Phys. Rev. B 41, 7906 (1990)

[24] M.J. Laughton et al., Phys. Rev. B 44, 1150 (1991)

[25] T. Ando, Phys. Rev. B 44, 8017 (1991)

[26] J.A. Brum, G. Bastard, in: S.P. Beaumont, C.M. Sotomajor Torres (eds.), Science and Engineering of One- and Zero-Dim. Semicond., Plenum Press, N.Y. (1990)

[27] P. Streda, J. Kucera, A.H. MacDonald, Phys. Rev. Lett. 59, 1973 (1987)

[28] L.I. Schiff, Quantum Mechanics, McGraw-Hill Book Company (1968)

[29] E. Castaño, G. Kirczenow, Phys.Rev.B 45, 1514 (1992) 